\title{
Uso y beneficios de la gamificación en la enseñanza de las matemáticas
}

\author{
Use and benefits of gamification in mathematics education \\ a José Omar Hernández-Peñaranda, banz Jaramillo-Benítez, ' Jaime Fernando Rincón-Leal \\ a*Docente, elpensador72@gmail.com, https://orcid.org/0000-0001-8017-3195, Institución educativa San Francisco de Sales, Cúcuta, Colombia \\ bMaestría en prácticas pedagógicas, j_jaramillo1@unisimon.edu.co, https://orcid.org/0000-0002-5988-2699, Institución Educativa Juan Bautista La Salle, Cúcuta \\ Colombia \\ 'Estudiante de Ingeniería Industrial, jaimefernandorlea@ufps.edu.co, https://orcid.org/0000-0003-4747-0360, Universidad Francisco de Paula Santander, Cúcuta, \\ Colombia
}

Forma de citar: Hernández-Peñaranda, J. O., Jaramillo-Benítez, J., \& Rincón-Leal, J. F. (2020). Uso y beneficios de la gamificación en la enseñanza de las matemáticas. Eco Matemático, 11 (2), 30-38

Recibido: 3 Marzo de 2020

Aceptado: 9 Abril 2020

\section{Palabras clave}

Gamificación; Aprendizaje;

Tic
Resumen:La presente investigación tuvo como objetivo realizar una revisión en diferentes bases de datos en español con el fin de identificar los restos y beneficios que tiene los juegos y la gamificación el ser incluidos en la aulas de clase , como estrategia didáctica y pedagógica para la enseñanza y el aprendizaje de las matemáticas, como se resultado se obtuvo, que para el docente realizar aulas innovadores con las Tecnología de la Información y la Comunicación (TIC), para el uso de la gamificación deber tener dedicación, ser estratégico y planear de manera adecuada su intervención, y para los alumnos este tipo de clase las percibe de manera positiva y su proceso de aprendizaje es satisfactorio, desarrollando así sus competencias y pensamientos matemáticos.

*Autor para correspondencia: elpensador72@gmail.com

Doi: https://doi.org/10.22463/17948231.3200 


\section{Keywords \\ Gamification; \\ Learning, \\ Tic}

\begin{abstract}
The aim of this research was to conduct a review in different databases in Spanish in order to identify the remains and benefits of games and gamification to be included in the classroom, as a didactic and pedagogical strategy for teaching and learning mathematics, as a result was obtained, that for the teacher to carry out innovative classrooms with Information and Communication Technology (ICT), for the use of gamification should have dedication, be strategic and adequately plan their intervention, and for students this type of class is perceived positively and their learning process is satisfactory, thus developing their skills and mathematical thinking.
\end{abstract}

\section{Introducción}

Durante mucho tiempo los estudiantes han tenido dificultad con el aprendizaje de las matemáticas, esto se debe de alguna forma en cómo los docentes enseñan sus conocimientos, es por ello que para nadie secreto que un gran porcentaje de los estudiantes en las instituciones educativas muestran en cierta forma resistencia a dejarse permear por la importancia que tiene en la vida práctica las matemáticas, las que inciden de forma directa en nosotros desde lo más elemental hasta lo más complejo.

De la misma manera, las matemáticas están en todo lo que hacemos, desde escribir las coordenadas de la casa donde vivimos, pasando por el peso y la talla en el proceso de crecimiento, los porcentajes de nutrientes en un plato de comida hasta llegar a los cálculos en la construcción de una obra de ingeniería, ellas están y estarán presentes en la vida de cada individuo.

Por lo tanto, en función de buscar diferentes estrategias didácticas Aristizábal, Colorado \& Gutiérrez (2016) afirman que el juego es la mejor alternativa para la enseñanza de las matemáticas en básica primaria el cual sirve para desarrollar el pensamiento numérico en las cuatro operaciones fundamentales, permitiendo potenciar las habilidades de cálculo y relaciones entre ellas , para familiarizarse y reafirmar el conocimiento de las operaciones : adicción, sustracción, multiplicación y cociente, teniendo en cuenta los factores como la sorpresa, la práctica, y de la misma manera se puede utilizar para la evaluación de dicho aprendizaje.

De la misma manera gran porcentaje de los docentes se han preguntado en sus aulas de clase ¿Cómo mejorar el aprendizaje de las matemáticas de forma divertida?, para ello el Alex Beard su libro otras formas de aprender (2019), afirma sobre propia práctica pedagógica que en la escuela donde trabaja: enseñaba con los métodos que utilizaba Sócrates en el ágora hace unos 2.000 años a unos niños que tenían sus celulares y vivían en el futuro, esta reflexión de Alex Beard es quizá una proyección de lo que ocurre con los docentes en general,ya que desconocen el poder que tienen hoy las nuevas metodologías, y la importación de saber e estilo de aprendizaje de los estudiantes (Jaramillo, Rincón y Rincón, 2020) y a la vez la capacidad motivadora que poseen , un ejemplo fehaciente en la actualidad es la gamificación bien estructurada y orientada según el estilo de aprendizaje.

Es importante antes de avanzar tener presente la influencia que ejerce en los niños y adolescentes el amplio universo de los videojuegos, es un hecho que la escuela entra día a día en una competencia invisible con los universos coloridos y dinámicos de este fenómeno que surgió un poco antes de la década de los 50 del siglo XX. 
En el 1940 aparece lo que se considera el primer videojuego presentado en la exhibición de Westinghouse durante la feria mundial de Nueva York, creado por el físico nuclear Edward Uhler Condon se trataba de un juego de matemáticas muy simple de persona VS ordenador. De ahí en adelante la industria de los videojuegos fue evolucionando de manera exponencial hasta el día de hoy con juegos de realidad virtual.

Es por ello que en la actualidad existen docentes que utilizan en el aula de manera exitosa los juegos más antiguos que incentivan además del aprendizaje las relaciones interpersonales mejorando de manera colaborativa los conceptos de diferentes áreas, un ejemplo de esto es el ajedrez, en el artículo Beneficios del ajedrez para los niños(4) menciona los estudios de la Universidad de Trier en Alemania, en uno de sus estudios llevado durante 4 años se compara a dos grupos de niños de primaria que se describen así: los autores del estudio quitaron a los alumnos de la línea A una hora semanal de matemáticas para dedicarla al ajedrez. En ese mismo periodo, los alumnos de la línea B siguieron recibiendo las tres horas semanales de clase de matemáticas que tenían previamente. Tras este largo periodo de análisis, los investigadores constataron que la nota en la asignatura de Matemáticas de los alumnos de la línea A siempre se situaba considerablemente por encima de la obtenida por los alumnos de la línea B. Con lo que concluyeron que el juego de ajedrez influye de manera directa en el desarrollo del razonamiento matemático.

Es por ello que cuando el docente enlaza su enseñanza disciplinar con la innovación creativa en el aula, no solo logra el mejoramiento en la evaluación de su asignatura, sino que adicionalmente logra encausar y motivar a sus estudiantes en buscar nuevos retos que prueben sus conocimientos indagando nuevas y mejores maneras de resolver problemas.
En este mismo sentido los niños niñas $\mathrm{y}$ adolescentes experimentan día a día un nuevos retos, ya a su vez alcanzan un nivel superior en diferentes video juegos que ejecutan, puesto que cuando se juega este tipo de video juegos se está aprendiendo una y otra vez de manera secuencial, ya que los juegos permiten reiniciar cuando se falla en el logro de una meta o de una misión, convirtiendo esta dinámica en un contante bucle de ensayo y error hasta que se domine su destreza y habilidad del mismo, sin duda alguna el combinar esta forma de ver el mundo virtual y digital de los video juegos en algo tan concreto como el aprendizaje de las matemáticas y de todas las asignaturas, no solo es alentador para el estudiante sino que le abre las posibilidades de un aprendizaje autónomo y secuencial.

\section{¿Qué es la gamificación?}

Según Gaitán (2013), La gamificación es una técnica de aprendizaje que traslada la mecánica de los juegos al ámbito educativo-profesional con el fin de conseguir mejores resultados: sirve para absorber conocimientos, para mejorar alguna habilidad y para recompensar acciones concretas, es por ello que cualquier actividad realizada en contexto de la gamificación busca lograr tres objetivos: la fidelización con el alumno, que se da al momento de crear un vínculo con el contenido trabajado.

Por otro lado, busca ser una herramienta motivadora y va en contra del aburrimiento, y finalmente, quiere optimizar y recompensar al alumno en aquellas tareas en las que no hay ningún incentivo más que el propio aprendizaje.

En otras palabras (Romero, Torres \& Aguaded, 2016) Afirman que gamificar no se trata de diseñar un juego, sino de aprovechar los sistemas de recompensas que usualmente tienen los juegos como: puntos, medallas, niveles, misiones, retos, logros, ventajas, así como las dinámicas y la estética para crear una experiencia que mantenga la atención y el interés en el desarrollo del contenido educativo. 
Es por ello que los docentes del siglo XXI deben usar el poder pedagógico de la gamificación que es sin duda un recurso valioso para seducir al estudiante en el aprendizaje de las matemáticas y al mismo tiempo aceptar el reto de innovación educativa, teniendo en cuanta que la gamificación requiere crear una narrativa que oriente el objetivo del proceso enseñanza-aprendizaje que se desea obtener en el aula

Tabla I. La relación de las dinámicas y mecánica de la gamificación, como elementos propios del juego (Ángel Torres-Toukoumidis y Luis M. Romero-Rodríguez, 2019)

$\begin{array}{llccc}\text { Puto } & \\ \text { Premios Estatus Logros } & \text { Competencia Altruismo } \\ & & \end{array}$

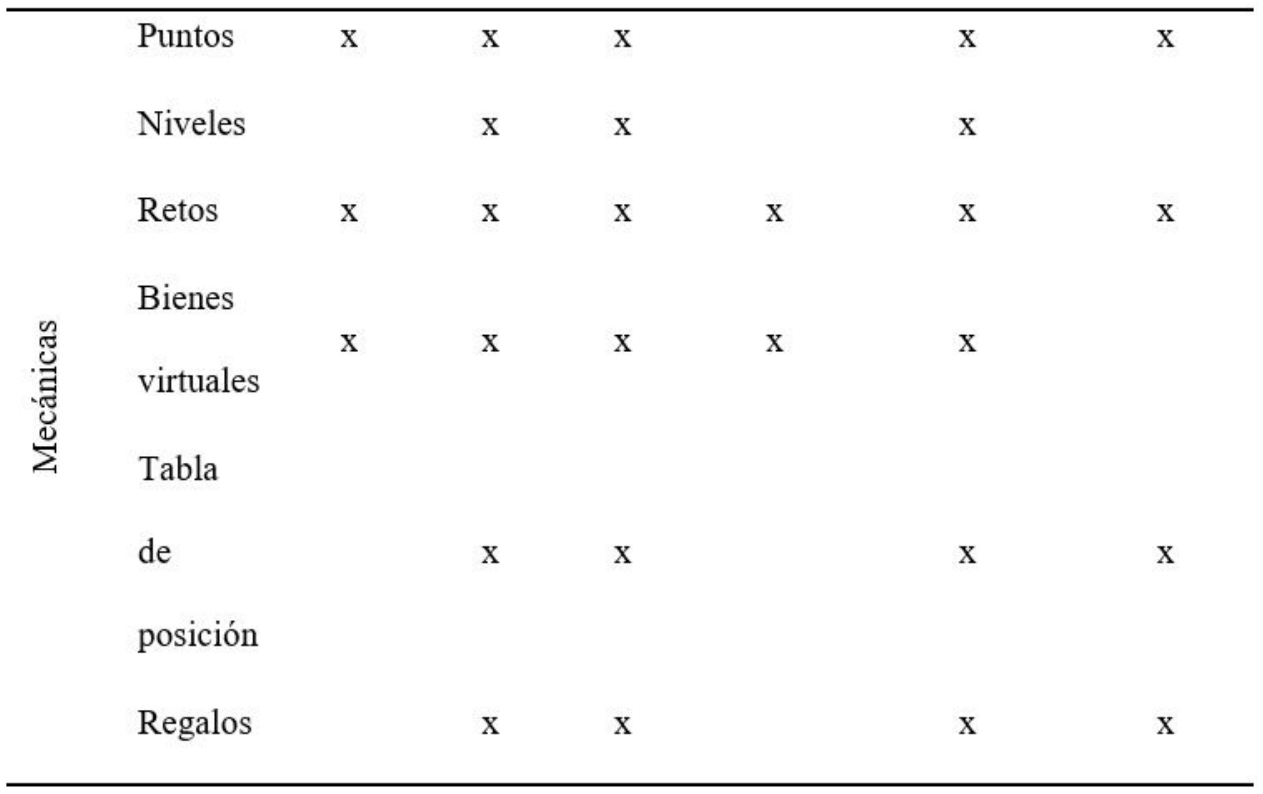

La Tabla I evidencia que las mecánicas son las estrategias a realizar, donde Ramírez (2014) las clasifica en básicas y accesorias. Las básicas, también conocidas como PBL (points, badges, leaderboards), son los puntos, las medallas y las clasificaciones. Dentro de las accesorias estarían los niveles, los bienes virtuales, los desafíos o retos, las misiones, los premios, los regalos, las recompensas, la customización o los cuadros de mando.

Las estructuras de los juegos son la forma en que los jugadores o usuarios interactúan con el juego, es decir, cómo se comporta el juego o proyecto, qué provoca en los usuarios y qué necesidades satisface. En definitiva: los deseos humanos, lo que le gusta a la gente o lo que la gente espera al interactuar en el juego. En otras palabras, las dinámicas son el concepto, y el diseño implícito del juego, las mecánicas son los procesos que provocan el desarrollo del juego y los componentes son las implementaciones específicas de las dinámicas y mecánicas. (Ortiz-Colón et al., 2018).

Morales Artero en el 2013 afirma que la gamificación aplicada al aprendizaje puede favorecer a que los alumnos disfruten mejor las actividades propuestas, y a su vez que ejerciten habilidades y destrezas de forma más práctica, contribuyendo así a que aumente la motivación intrínseca.

Por otro lado, Ortegón y Emilia (2016) dicen que una propuesta de Gamificación en las matemáticas en la enseñanza del valor posicional 
de cantidades surge a partir de una experiencia personal de búsqueda tras la necesidad de encontrar nuevas formas de enseñar para el beneficio de los estudiantes.

\section{Los beneficios de la gamificación}

Al aprovechar la disposición natural que tienen niños, niñas y adolescentes para sumergirse en el mundo de los juegos o video juegos, es fácil entender que en las áreas como las matemáticas esto trae varios beneficios, entre los que se puede ser el centro del juego, la toma de sus propias decisiones, sentir que progresan, asumir nuevos retos, participar en un entorno social abierto, tener reconocidos por sus logros y recibir retroalimentación inmediata, y sobre todo que se puede ser utilizada en diferentes contextos (Borrás Gené, 2015).

Cada vez es común encontrar artículos y proyectos relacionados sobre la gamificación en la enseñanza de las matemáticas, una de ellas es Iza Salazar (2019) que usa la gamificación como estrategia innovadora para la enseñanza de las matemáticas en educación primaria, recopila en una tabla varias referencias que dejan pre ver que cada día los docentes en el área de matemáticas ven los beneficios de introducir dicha práctica en su quehacer pedagógico. 
Tabla II. Revisión de uso de la Gamificación revisada y mejorada por Bello, D. Cesar Mauricio y Hernández, P. José Omar (2018)

\begin{tabular}{|c|c|c|c|c|}
\hline Año & Base de Datos & Tema & País & Nombre de la Revista \\
\hline 2018 & Dialnet & $\begin{array}{l}\text { Math Mystery Box: Gamificando el } \\
\text { aprendizaje de las matemáticas. }\end{array}$ & España & $\begin{array}{l}\text { Revista de didáctica de las } \\
\text { matemáticas. }\end{array}$ \\
\hline 2018 & Dialnet & $\begin{array}{l}\text { El Ministerio de Robin Hood: una } \\
\text { experiencia de gamificación. }\end{array}$ & España & Revista Pensamiento matemático. \\
\hline 2016 & Google académico & $\begin{array}{l}\text { Integración curricular de una plataforma } \\
\text { online para el aprendizaje de las } \\
\text { matemáticas en educación primaria. }\end{array}$ & España & $\begin{array}{l}\text { Revista de edmetic Revista de } \\
\text { educación mediática y tic }\end{array}$ \\
\hline 2016 & Google académico & $\begin{array}{l}\text { El rol del juego digital en el aprendizaje } \\
\text { de las matemáticas: experiencia } \\
\text { conjunta en escuelas de básica primaria } \\
\text { en Colombia y Brasil. }\end{array}$ & Argentina & $\begin{array}{l}\text { Revista Electrónica de } \\
\text { investigación en educación en } \\
\text { ciencias }\end{array}$ \\
\hline 2019 & ProQuest & $\begin{array}{l}\text { Inteligencias múltiples y videojuegos: } \\
\text { Evaluación e intervención con software } \\
\text { TOI. }\end{array}$ & España & $\begin{array}{l}\text { Revista científica } \\
\text { educomunicación. }\end{array}$ \\
\hline 2018 & Scielo & $\begin{array}{l}\text { Gamificación en educación: una } \\
\text { panorámica sobre el estado de la } \\
\text { cuestión. }\end{array}$ & Brasil & Revista Educação e Pesquisa \\
\hline 2015 & Scielo & $\begin{array}{l}\text { Diseño e implementación de una } \\
\text { estrategia de gamificación en una } \\
\text { plataforma virtual de educación. }\end{array}$ & Bolivia & Revista Fides et Ratio \\
\hline 2016 & Redalyc & $\begin{array}{l}\text { Juegos digitales y gamificación } \\
\text { aplicados en el ámbito de la educación. }\end{array}$ & España & $\begin{array}{l}\text { RIED. Revista Tberoamericana } \\
\text { de Educación a Distancia }\end{array}$ \\
\hline 2017 & Redalyc & $\begin{array}{l}\text { Tecnologías y nuevas tendencias en } \\
\text { educación: aprender jugando. El caso de } \\
\text { Kahoot. }\end{array}$ & España & $\begin{array}{l}\text { RIED. Revista Iberoamericana } \\
\text { de Educación a Distancia. }\end{array}$ \\
\hline 2017 & Redalyc & $\begin{array}{l}\text { Los videojuegos como medio de } \\
\text { aprendizaje: Un estudio de casos en }\end{array}$ & España & $\begin{array}{l}\text { RIED Revista iberoamericana de } \\
\text { educación a distancia. Revista }\end{array}$ \\
\hline
\end{tabular}

La Tabla II evidencia el uso de la gamificación en la educación con el uso de los videojuegos para mejorar el proceso de enseñanza y aprendizaje en las aulas innovadoras

\section{Herramientas para gamificar en matemáticas}

Muchos docentes han escuchado de esta tendencia en educación, sin embargo, no se animan por que desconocen cómo hacerlo o por dónde empezar. Lo que se debe saber es que hay un gran abanico de posibilidades que se pueden explorar para poder iniciar experiencias realmente significativas especialmente en el área de matemáticas. 
Tabla III. Plataformas para el uso de la Gamificación

\begin{tabular}{|c|c|}
\hline Nombre $y$ link de acceso & Uso \\
\hline Knowre / https-//www.knowre.co & $\begin{array}{l}\text { Esta plataforma utiliza la opción de los videojuegos para aprender } \\
\text { matemáticas de manera divertida, según sus creadores los estudiantes no } \\
\text { odian las matemáticas, odian sentirse frustrados. Por eso esta plataforma } \\
\text { es una muy buena opción para trabajar retos de algebra y geometria }\end{array}$ \\
\hline MyClassGame' https://www.myclassgame.es/ & $\begin{array}{l}\text { Esta plataforma ayuda a los docentes a combinar la gamificación con el } \\
\text { aprendizaje basado en proyectos y aprendizaje cooperativo, se puede } \\
\text { usar en cualquier nivel educativo, además gi se accede desde una cuenta } \\
\text { de Google permite articularse con Google Classroom. }\end{array}$ \\
\hline Brainscape / https $/ /$ www brainscape.com $/$ & $\begin{array}{l}\text { Esta es una plataforma basada en flashcards o tarjetas didácticas, en este } \\
\text { género es la plataforma con la mayor cantidad de flashcards que incluye } \\
\text { un variopinto temario que te permitirá abordar cualquier tipo de } \\
\text { temática. }\end{array}$ \\
\hline & $\begin{array}{l}\text { Esta plataforma tiene su origen y creación en España, pero con un alto } \\
\text { indice de uso a lo largo de todo el mundo, tiene la opción de crear juegos }\end{array}$ \\
\hline Cerebriti / https://www.cerebriti.com/ & $\begin{array}{l}\text { por parte de los estudiantes y de jugar los creados por sus profesores, } \\
\text { todo esto con el objetivo de afianzar los conocimientos adquiridos en el } \\
\text { aula. }\end{array}$ \\
\hline & $\begin{array}{l}\text { Inicialmente Minecraft se creó como un juego más para la diversión, sin } \\
\text { embargo, la edición de educación de Minecraft es un ejemplo de como }\end{array}$ \\
\hline $\begin{array}{l}\text { Minecraft Education Edition / } \\
\text { https//education minecraft.net/es-es/ homepage }\end{array}$ & $\begin{array}{l}\text { se logra usar el juego para fínes didácticos. La única limitación es la } \\
\text { creatividad de los docentes, se puede usar en infinidad de tema, es una } \\
\text { plataforma que engancha fácilmente a los estudiantes de cualquier nivel } \\
\text { educativo. }\end{array}$ \\
\hline Classcraft / https://www.classcraft.com/es-es/ & $\begin{array}{l}\text { la plataforma más cercana a lo que es un videojuego con fines } \\
\text { educativos. Su interfaz es tremendamente visual y atractiva que permite } \\
\text { crear personajes que deberán cooperar para ir ganando recompensas que } \\
\text { les permitirán ir subiendo de status. Y a la hora de evaluar también } \\
\text { podemos usar plataformas tipo freemium, es decir plataformas gratuitas, } \\
\text { pero con opciones de pago que permitirán aprovechar al } 100 \% \text {. }\end{array}$ \\
\hline Quizizz / https:/quizizz.com/ & $\begin{array}{l}\text { La plataforma permite que los docentes reutilicen preguntas diseñadas } \\
\text { en sus procesos particulares. Esto permite sumergir al estudiante en una } \\
\text { competencia con los demás compañeros, potencia el deseo de aprender } \\
\text { y acertar cada reto propuesto por el profesor. }\end{array}$ \\
\hline & $\begin{array}{l}\text { Es una herramienta que permite crear juegos de preguntas y respuestas } \\
\text { de forma muy }\end{array}$ \\
\hline Kahoot / https:/lkahoot.com/ & $\begin{array}{l}\text { Es muy similar a Quizziz, ya que le permite crear un verdadero ambiente } \\
\text { de competencia ludificado para afianzar los conocimientos adquiridos, } \\
\text { se puede usar como diagnóstico, seguimiento o finalización de un tema } \\
\text { propuesto. }\end{array}$ \\
\hline
\end{tabular}


La Tabla III evidencia la importancia y el uso de la Gamificación la cual tiene como objetivo incrementar la motivación, fomentar el compañerismo y aplicar los contenidos aprendidos en cualquier otro contexto no relacionado a los juegos, llegando a ser su aprendizaje significativo y valioso

\section{Conclusiones}

Frente a la realidad actual de la educación es imperante que los docentes asuman retos que involucren nuevas metodologías que faciliten la interiorización de los contenidos dentro y fuera del aula, los docentes de Matemáticas tienen a su disposición una serie de herramientas sobre la gamificación que pueden usar para hacer más atractivos el aprendizaje del área.

Es por ello la gamificación se puede conjugar con otras metodologías que involucren las TIC, haciendo que el aprendizaje se realice en periodos sincrónicos y asincrónicos en el momento de Gamificar el contenido del área de matemáticas es necesario que el docente se involucre y explote su creatividad pedagógica y didáctica, lo que dará como resultado, altos niveles de efectividad, además que mejora la creatividad, la motivación y evita la frustración frente a los contenidos propuestos.

\section{Referencias}

Aristizábal Z , Jorge. Colorado T , Hernán Humberto, ., \& Gutiérrez Z, H. (2016). El juego como una estrategia didáctica para desarrollar el pensamiento numérico en las cuatro operaciones básicas. Sophia, 12, 118. https://www.redalyc. org/pdf/4137/413744648009.pdf

Beard, A., \& Hermida Lazcano, P. (2019). Otras formas de aprender : qué funciona en educación y por qué (Plataforma Editorial (Ed.); Vol. 1). Plataforma Editorial
Bello Díaz, C. M., \& Hernández Peñaranda, J. O. (2018). Acordemos: desarrollo de competencias ciudadanas para la gestión de conflictos, a través de una propuesta pedagógica mediada por las TIC. Universidad Autónoma de Bucaramanga, 1(1), 16. https://repository.unab.edu.co/ handle/20.500.12749/2490

Borras Gene, O. (2015). Fundamentos de la gamificación Universidad Politécnica de Madrid. Gabinete de Tele-Educación. Universidad Politécnica de Madrid, 33. http://oa.upm. es/35517/1/fundamentos de la gamificacion_ v1_1.pdf

Gaitán, V. (2013). Gamificación: el aprendizaje divertido | Educativa. Educativa. https://www. educativa.com/blog-articulos/gamificacion-elaprendizaje-divertido/

Iza Salazar, M. M. (2019). La Gamificación como estartegia innovadora para la enseñanza de las matemáticas en educación primaria. Pontificia Universidad Católica Del Ecuador, 1(1), 41. http://repositorio.puce.edu.ec/ handle/22000/17868

Jaramillo, J. E., Rincon Leal, J. F., \& Rincon Leal, O. L. (2020). Impact of learning styles on multiple intelligences in first semester math students. Journal of Physics: Conference Series, 1645(1). https://doi.org/10.1088/17426596/1645/1/012015

Morales, J. (2013). La gamificación en la universidad para mejorar los resultados académicos de los alumnos. Quinto Congreso Virtual Iberoamericano de Calidad En Educación Virtual y a Distancia, 1-15. http://eprints.uanl. mx/8087/1/m8_2.pdf

Nueve bolas de helado por la dama | ChessBase. (n.d.). Retrieved July 24, 2021, from https:// es.chessbase.com/post/nueve-bolas-de-helado- 
por-la-dama

Ortegón Yánez, M. E. (2016). Gamificación de las matemáticas en la enseñanza del valor posicional de cantidades. Universidad Internacional de La Rioja (UNIR), 55. https://reunir.unir.net/ bitstream/handle/123456789/4704/ORTEGON YAÑEZ\%2C MARTHA EMILIA_Censurado. pdf? sequence $=1 \&$ isAllowed $=\mathrm{y}$

Ortiz Colón, Ana. Jordan, Juan. Agredal, M. (2018). Gamificación en educación: una panorámica sobre el estado de la cuestión. Scielo, 1, 14. https://doi.org/https://doi.org/10.1590/S16784634201844173773

Ramiréz, J. L. (2014). GAMIFICACIÓN. Mecánicas de juegos en tu vida personal y profesional (Sclibro (Ed.)).

Torres Toukoumidis, Angel . Rodríguez, Luis Romero. Rodríguez, Amor Pérez. Björk, S. (2019). Modelos de calidad de las plataformas de recomendación de aplicaciones infantiles y juveniles. Ocnos. Revista de Estudios Sobre Lectura, 18(2), 17-30. https://doi.org/10.18239/ ocnos 